\title{
BMJ Open Barriers to the implementation of sexual and reproductive health education programmes in low-income and middle- income countries: a scoping review protocol
}

\author{
Moreblessing Chipo Mashora (D) , ${ }^{1}$ Tafadzwa Dzinamarira (D) ,,3 \\ Claude Mambo Muvunyi ${ }^{2,3}$
}

To cite: Mashora MC, Dzinamarira T, Muvunyi CM. Barriers to the implementation of sexual and reproductive health education programmes in low-income and middleincome countries: a scoping review protocol. BMJ Open 2019;9:e030814. doi:10.1136/ bmjopen-2019-030814

\section{- Prepublication history for} this paper is available online. To view these files, please visit the journal online (http://dx.doi. org/10.1136/bmjopen-2019030814).

Received 03 April 2019 Revised 19 August 2019 Accepted 12 September 2019

D) Check for updates

(c) Author(s) (or their employer(s)) 2019. Re-use permitted under CC BY-NC. No commercial re-use. See rights and permissions. Published by BMJ.

${ }^{1}$ Public Health, Mount Kenya University, Kigali, , Rwanda ${ }^{2}$ College of Medicine and Health Sciences, University of Rwanda, Kigali, Rwanda

${ }^{3}$ ICAP, Mailman School of Public Health, Columbia University, Kigali, Rwanda

Correspondence to Tafadzwa Dzinamarira; anthonydzina@gmail.com

\section{ABSTRACT}

Introduction Health education programmes (HEPS) have been associated with a number of benefits. These include providing individuals with information on matters related to their mental, social, physical as well as emotional health. HEPs also play a major role in preventing diseases and reducing the level of engagement of individuals in risky behaviours. While this is the case, there are barriers to the effective implementation of HEPs, especially in low-income and middle-income countries (LMICs) where resources are scarce. Available evidence has revealed socioeconomic challenges ranging from literacy issues, discomfort about issues of sexuality, and cultural barriers to financial constraints as key barriers to the implementation of sexual and reproductive health HEPs in LMICs. We will focus on HEPs related to sexual and reproductive health; all age groups will be considered with no restrictions on geographical setting nor model of HEP delivery. This review will map literature on the barriers to the effective implementation of HEPs in LMICs to guide future implementation research.

Methods Arksey and 0'Malley's 2005 scoping methodological framework will act as the guide for this review. We will search the following electronic databases: EBSCOhost (Academic search complete, PsycINF0, Health Sources, CINAHL and MEDLINE with full text), Google Scholar, PubMed, SCOPUS, Science Direct and Web of Science. Grey literature from Mount Kenya University theses and dissertations, governments' as well as international organisations' reports, such as WHO, and reference lists of included studies will be searched for eligible studies. We will limit our search to publications from 1 January 2000 to 30 September 2019. Using thematic content analysis, we will employ NVivo V.12 to extract the relevant outcomes from the included articles. We will conduct a quality appraisal of the included articles using the mixed methods appraisal tool (MMAT) version 2018.

Ethics and dissemination No ethical approval is needed for the study as it will not include animal nor human participants. The results of the proposed scoping review will be disseminated electronically, in print and through conference presentation as well as at key stakeholder meetings.

\section{Strengths and limitations of the study}

- The results of this review will establish a baseline understanding of barriers to the implementation of sexual and reproductive health education programmes in low-income and middle-income countries and will discuss potential strategies to ensure effective implementation.

- Here, we propose the use of an established scoping review methodology with a comprehensive search strategy that includes grey literature.

- The study will conduct a formal quality assessment of included studies guided by an established mixed methods appraisal tool.

- A limitation of this review is its potential to miss relevant articles, given that the findings will be limited to articles written in English.

\section{BACKGROUND}

One of the Sustainable Development Goals set forth by the United Nations for 2030 is ensuring healthy lives and promoting wellbeing for all, at all ages. ${ }^{1}$ Health should be promoted through the provision of a decent living standard, education, good labour conditions, physical exercise, and means of rest and recreation. ${ }^{2}$ Historically, health education programmes (HEPs) are aimed at providing the requisite skills and competencies to ensure that people are in a good position to embrace healthy behaviours voluntarily. ${ }^{2}$ HEPs have been shown to ensure the prevention of illness and improve outcomes among different populations. ${ }^{2-5}$

Available literature shows benefits linked to HEPs through increases in the skills and knowledge of individuals on matters related to health. ${ }^{6}$ HEPs have been used to provide individuals with information concerning mental health, social health, physical health 
as well as emotional health. ${ }^{7-9}$ The other notable benefits that are associated with HEPs include the prevention of diseases and the reduction of the level of engagement of individuals in highly risky behaviours. ${ }^{10}{ }^{11}$ When there are well-implemented HEPs, there can be various positive changes in behaviour. This has been shown to have the ability to significantly lower the risks that are associated with alcohol, tobacco as well as other types of drugs. ${ }^{6-11}$

Various scholars have investigated the benefits of HEPs for sexual and reproductive health. Poor healthseeking behaviour is a major factor leading to preventable morbidity and mortality. HEPs for sexual and reproductive health implemented in Uganda, Kenya and Zimbabwe have reported improved health-seeking behaviour as an outcome. ${ }^{12-15}$ In Uganda, improved health-seeking behaviour resulted in an improved uptake of HIV services ${ }^{12}$ and an improved attendance to clinic appointments or routine check-ups. ${ }^{13}$ Similar findings were reported in Kenya and Zimbabwe. ${ }^{14}{ }^{15}$ Further, a notable decrease in risky sexual behaviour was also reported. ${ }^{12}{ }^{15}$ In the context of couples, improved accompanying of partners to health services and improved communication with partners on health issues have been reported as notable benefits of HEPs in sexual and reproductive health. ${ }^{12} 13$

While HEPs have a number of benefits linked to them, there are various challenges that hinder the effective implementation of HEPs in low-income and middle-income countries (LMICs). To the researchers' best knowledge, there is no published scoping review reporting evidence on barriers to the implementation of HEPs in LMICs. We will use a scoping review to map literature on reported barriers to the effective implementation of HEPs in LMICs, with the aim of guiding future research to provide strategies that can be used to counter such barriers.

\section{METHODS AND ANALYSIS}

Through systematic searching, selection and synthesis of existing knowledge, a scoping review will be used for synthesising available evidence. Arksey and O'Malley's framework, ${ }^{16}$ with modifications from Levac et al, ${ }^{17}$ will guide this review. This framework involves identifying the research questions; identifying the relevant studies; selecting the studies to be used during the research process; charting the data; and then ensuring that the results are collated, summarised, and reported effectively and efficiently. The review will adopt the mixed methods appraisal tool, $2018,{ }^{18}$ for the assessment of the quality of included articles. For thematic content analysis, we will use NVivo V. $12^{19}$ for the extraction of relevant outcomes. Included studies will be uploaded into NVivo V.12 and information on barriers to the implementation of sexual and reproductive health HEPs will be coded. We will report our findings on the number of studies retrieved from the search and the total number of studies excluded at each screening stage guided by the PRISMA Extension for Scoping Reviews, ${ }^{20}$ as outlined in figure 1 .

We chose to use the scoping methodology as it has been reported to be a useful approach for determining the need for and value of a future primary study or a full systematic review. ${ }^{16}$ Further, scoping reviews allow a broad overview of the evidence pertaining to a topic, in order to clarify key concepts and identify gaps. ${ }^{16}$ We anticipate that our scoping review will be used to identify a topic area for a future systematic review.

\section{Eligibility of research}

In order to determine the eligibility for the research question, the Population, Concept and Context tool will be used. This is demonstrated in table 1 .

Our research question is: What is the evidence on barriers to the implementation of HEPs on sexual and reproductive health in LMICs?

\section{Identification of relevant studies}

The researchers will carry out a comprehensive search of articles published between 2000 and 2019. We will search the following electronic databases: EBSCOhost (Academic search complete, PsycINFO, Health Sources, CINAHL and MEDLINE with full text), Google Scholar, PubMed, SCOPUS, Science Direct and Web of Science. In addition, we will search grey literature from Mount Kenya University theses and dissertations, and governments' as well as international organisations' reports, such as WHO, for eligible studies. Finally, we will search reference lists of included studies for eligible articles. The search terms to be used will be (low[All Fields] AND middle[All Fields] AND ("income" [MeSH Terms] OR "income"[All Fields]) AND countries[All Fields]) AND sexual AND reproductive AND ("health promotion"[MeSH Terms] OR "health education promotion"[All Fields]) AND ("'health education" [MeSH Terms] OR ("health"[All Fields] AND "education"[All Fields]) OR "health education"[All Fields]) AND programs[All Fields]) AND "challenges"[All Fields] OR barriers[tiab] OR barrier[tiab] OR impeding[tiab] OR hindering[tiab].

The search strategy will be adapted to every database. Every search will be documented in detail, illustrating the keywords, the date when the search was performed, the search engine as well as the number of publications that were retrieved. We conducted a pilot search to assess the feasibility of answering the research question by a scoping review. Results are presented in table 2.

\section{Study selection}

We will search for primary studies, including randomised controlled trials, non-randomised controlled trials and observational studies, that report evidence on barriers to the implementation of HEPs in LMICs. We developed selection criteria to make sure that all the relevant studies on the barriers to the implementation of sexual and reproductive HEPs in LMICs are included. 


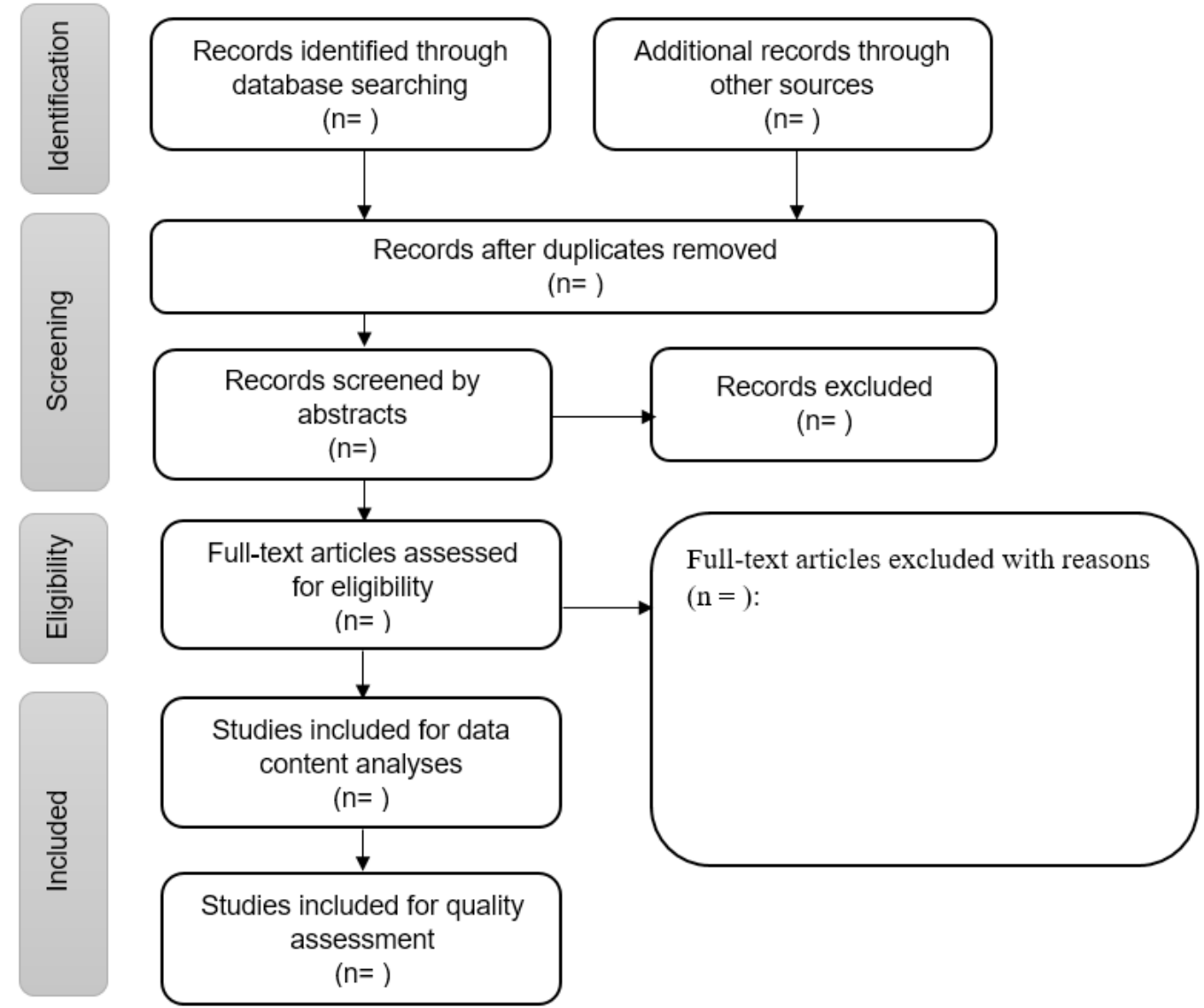

Figure 1 PRISMA Extension for Scoping reviews, 2018 flow diagram.

\section{Inclusion criteria}

In order for a study to be included in the review, the following set of criteria must be met:

1. Reporting evidence on barriers to the implementation of sexual and reproductive health education programmes in LMICs.

2. Studies published in English.

3. Studies published between 1 January 2000 and 30 September 2019.

Table 1 Framework for determining eligibility for the research question

Human participants in low-income and middle-income countries, as classified by the World Bank at the time of the Population publication of the article

$\begin{array}{ll}\text { Concept } & \begin{array}{l}\text { Reported barriers to the implementation } \\ \text { of HEPs. For the purposes of this review, } \\ \text { health education will be defined as the } \\ \text { provision of health information and } \\ \text { knowledge to people and communities }\end{array} \\ \text { Context } & \text { Sexual and reproductive health }\end{array}$

HEP, health education programme.

\section{Charting the data}

The titles of the studies, their abstracts as well as the full texts of all publications will be identified through searches for eligible publications. The reviewers will evaluate the titles, abstracts and then the full texts of all publications identified through searches for potentially eligible publications. Study selection will occur in three stages. As the first stage, one reviewer will screen the titles from the databases guided by the eligibility criteria. Following title screening, two reviewers will screen abstracts. Discrepancies in reviewers' responses at the abstract screening stage will be resolved through a discussion until consensus is reached. Finally, two reviewers will review full-text articles. Discrepancies in reviewers' responses at the full article screening stage will be resolved by involving a third screener. Information will be extracted from the included articles using the data charting form (box 1). The form will be constantly updated in an iterative process according to the data extracted and in order to fully answer the research question. The PRISMA chart (figure 1) will be used to document the screening results.

\section{Collating, summarising and reporting results}

In line with Arksey and O'Malley's framework, ${ }^{16}$ the researchers will extract data that are linked to the research 
Table 2 Results from pilot search in PubMed

\begin{tabular}{|c|c|c|c|}
\hline Keywords search & $\begin{array}{l}\text { Date of } \\
\text { search }\end{array}$ & $\begin{array}{l}\text { Search } \\
\text { engine used }\end{array}$ & $\begin{array}{l}\text { Number of publications } \\
\text { retrieved }\end{array}$ \\
\hline $\begin{array}{l}\text { (low(All Fields)AND middle(All Fields)AND ("income"(MeSH Terms)OR } \\
\text { "income"(All Fields)) AND countries(All Fields)) AND sexual AND reproductive } \\
\text { AND ("health promotion"(MeSH Terms)OR "health education promotion"(All } \\
\text { Fields)) AND (("health education"(MeSH Terms)OR ("health"(All Fields)AND } \\
\text { "education"(All Fields)) OR "health education"(All Fields)) AND programs(All } \\
\text { Fields)) AND "challenges"(All Fields)OR barriers(tiab)OR barrier(tiab)OR } \\
\text { impeding(tiab)OR hindering(tiab) }\end{array}$ & $\begin{array}{l}15 \text { August } \\
2019\end{array}$ & PubMed & 234338 \\
\hline
\end{tabular}

objectives through the use of a thematic content analysis approach. We will employ NVivo V.12 for thematic content analysis. The reviewers will analyse the full-text articles for reported evidence on barriers to the implementation of sexual and reproductive health HEPs in LMICs. All data pertaining to barriers to the implementation of HEPs in LMICs will be extracted from the included articles. This will be done through coding. Finally, an overall interpretation will be performed regarding how the thematic areas relate to one another, explaining how the various concepts relate to the research question.

\section{Quality appraisal}

We will employ the mixed methods appraisal tool $(\text { MMAT })^{18}$ for evaluating the quality of the articles that have been selected to be included in the study. ${ }^{18}$ Through the use of the tool, the researchers will be in a position to evaluate the aim of the study, the methodology used, the study design used, the recruitment of the research participants, the collection of data, the manner in which analysis of data took place, the presentation of the study findings, and the authors' discussions and conclusions. The MMAT has been shown to be useful for appraising the quality of primary research. ${ }^{18}$ Critical appraisal entails making judgements; hence, there will be two independent reviewers who will be involved during the process. For every study included, there will be criteria that will be used for appraisal. The overall percentage quality score will be calculated. For the purposes of this review, scores of $\leq 50 \%$ will be considered as low quality, while a score in the range of $51 \%-75 \%$ will be regarded as average quality. A score in the range of $76 \%-100 \%$ will be considered

\section{Box 1 Data charting form}

- Author and publication year.

- Study title.

- Total participants.

Age of participants.

- Geography of the study (where was the study area).

- Type of sexual and reproductive health education programme (HEP) carried out.

- Details on the barriers to the implementation of HEPs.

> Study design.

Most relevant findings.

Conclusions. as high quality. Reviewers will follow the scoring guide provided in MMAT version 2018. ${ }^{18}$

\section{Ethical considerations}

No ethical approval is needed for the study because it will not include animal nor human participants.

\section{Patient and public involvement}

This study does not involve patients or the public.

\section{DISCUSSION}

LMICs generally experience a number of healthcare challenges. ${ }^{10}$ As Iqbal $e t a l^{21}$ point out, one of the main challenges faced is the inequity that exists when it comes to access to healthcare across the globe, and in particular in LMICs. Much focus has been placed on improving access to health services through improving healthcare systems. However, limited studies have been carried out to explore barriers to the implementation of these interventions.

In the context of sexual and reproductive health, HEPs have been documented to enhance the health of various population groups and to be effective in improving health outcomes in relation to sexually transmitted diseases. ${ }^{22-24}$ Vanwesenbeeck et al reported that HEPs for sexual and reproductive health among adolescents and young boys resulted in improved knowledge and skillsets essential for safer sexual choices. ${ }^{25}$ This calls for the need for HEPs to improve health outcomes.

The scoping review will include evidence published from 2000 to 2019. The studies that will be included in the review will contain information concerning barriers to the implementation of HEPs in LMICs. The researchers anticipate the obtainment of relevant studies that report on the barriers to the implementation of HEPs in LMICs. The findings of this review will be highly beneficial to policy-makers who are involved when HEPs are being crafted to improve health outcomes. In addition, the findings of the review will help design strategies that can be used to ensure successful implementation of HEPs in LMICs. Researchers interested in carrying out further research in the area can also use the findings of the study as a basis for their research. The review will be disseminated electronically and in print, and it will be presented at scientific conferences on health education promotion. 
Acknowledgements The authors would like to extend their appreciation to the UKZN Systematic Review Services, School of Nursing and Public Health (SNPH), College of Health Sciences, University of KwaZulu-Natal for technical training on conducting scoping reviews.

Contributors MCM conceptualised the study and prepared the draft proposal. MCM and TD contributed to the development of the background and planned the output of the research as well as the design of the study. MCM and TD prepared the manuscript, and CMM critically reviewed it. All authors (MCM, TD and CMM) contributed to the reviewed draft version of the manuscript and approved the final version.

Funding The authors have not declared a specific grant for this research from any funding agency in the public, commercial or not-for-profit sectors.

Competing interests None declared.

Patient consent for publication Not required.

Provenance and peer review Not commissioned; externally peer reviewed.

Open access This is an open access article distributed in accordance with the Creative Commons Attribution Non Commercial (CC BY-NC 4.0) license, which permits others to distribute, remix, adapt, build upon this work non-commercially, and license their derivative works on different terms, provided the original work is properly cited, appropriate credit is given, any changes made indicated, and the use is non-commercial. See: http://creativecommons.org/licenses/by-nc/4.0/.

\section{ORCID iDs}

Moreblessing Chipo Mashora https://orcid.org/0000-0002-8242-0169

Tafadzwa Dzinamarira http://orcid.org/0000-0002-9929-5739

\section{REFERENCES}

1 Lee BX, Kjaerulf F, Turner S, et al. Transforming our world: implementing the 2030 agenda through sustainable development goal indicators. J Public Health Policy 2016;37:13-31.

2 Lobo R, Petrich M, Burns SK. Supporting health promotion practitioners to undertake evaluation for program development. $B M C$ Public Health 2014;14:1315.

3 Coe G, de Beyer J. The imperative for health promotion in universal health coverage. Glob Health Sci Pract 2014;2:10-22.

4 Kumar S, Preetha GS. Health promotion: an effective tool for global health. Indian J Community Med 2012;37:5-12.

5 Cameron KA, Schneider EC. Benefits of evidence-based health promotion/disease prevention programs for older adults and community agencies. Innov Aging 2018;2.

6 Darlington EJ, Violon N, Jourdan D. Implementation of health promotion programmes in schools: an approach to understand the influence of contextual factors on the process? BMC Public Health 2018;18:163.

7 Gucciardi E, Cameron JI, Liao CD, et al. Program design features that can improve participation in health education interventions. BMC Med Res Methodol 2007;7:47.
8 Cancelliere C, Cassidy JD, Ammendolia C, et al. Are workplace health promotion programs effective at improving presenteeism in workers? A systematic review and best evidence synthesis of the literature. BMC Public Health 2011;11:395.

9 Backman DR, Kohatsu ND, Paciotti BM, et al. Health promotion interventions for low-income Californians through Medi-Cal managed care plans, 2012. Prev Chronic Dis 2015;12:E196.

10 Petrakova A, Sadana R. Problems and progress in public health education. Bull World Health Organ 2007;85:963-5. discussion 96670.

11 Varming A, Torenholt R, Møller B, et al. Addressing challenges and needs in patient education targeting hardly reached patients with chronic diseases. Indian J Endocrinol Metab 2015;19:292-5.

12 Ghanotakis E, Hoke T, Wilcher R, et al. Evaluation of a male engagement intervention to transform gender norms and improve family planning and HIV service uptake in Kabale, Uganda. Glob Public Health 2017;12:1297-314.

13 Kabagenyi A, Ndugga P, Wandera SO, et al. Modern contraceptive use among sexually active men in Uganda: does discussion with a health worker matter? BMC Public Health 2014;14:286.

14 Odeny TA, Bailey RC, Bukusi EA, et al. Text messaging to improve attendance at post-operative clinic visits after adult male circumcision for HIV prevention: a randomized controlled trial. PLOS One 2012;7:e43832.

15 Chirau T. Prevalence and factors associated with risky sexual behaviour after male circumcision among clients circumcised at Spilhaus clinic in Harare.

16 Arksey H, O'Malley L. Scoping studies: towards a methodological framework. Int J Soc Res Methodol 2005;8:19-32.

17 Levac D, Colquhoun H, O'Brien KK. Scoping studies: advancing the methodology. Implementation Science 2010;5.

18 Hong QN, Pluye P, FÀbregues S, et al. Mixed methods appraisal tool (MMAT), version 2018. Canada, 2018.

19 Anon. Buy NVivo Now | NVivo. [online] Qsrinternational.com, 2019. Available: https://www.qsrinternational.com/nvivo/nvivo-products [Accessed 31 Mar 2019].

20 Tricco AC, Lillie E, Zarin W, et al. PRISMA extension for scoping reviews (PRISMA-ScR): checklist and explanation. Ann Intern Med 2018;169:467-73.

21 Iqbal U, Rabrenovic M, Li Yu-Chuan (Jack). Health care quality challenges in low- and middle-income countries. Int J Qual Health Care 2019;31:mzz031.

22 Barker G, Ricardo C, Nascimento M, et al. Engaging men and boys in changing gender-based inequity in health: evidence from programme interventions 2007.

23 Kirby D, Laris B. Effective curriculum-based sex and STD/HIV education programs for adolescents 2009;3:21-9.

24 Morales A, Garcia-Montaño E, Barrios-Ortega C, et al. Adaptation of an effective school-based sexual health promotion program for youth in Colombia. Soc Sci Med 2019;222:207-15.

25 Vanwesenbeeck I, Westeneng J, de Boer T, et al. Lessons learned from a decade implementing Comprehensive Sexuality Education in resource poor settings: The World Starts With Me. Sex Educ 2016;16:471-86. 